\title{
Avaliação da contaminação do carbofuran nos solos do Distrito de Irrigação do Gorutuba
}

\author{
Edson de O. Vieira ${ }^{1}$, Hélio T. Prates ${ }^{2}$, José R. B. Pereira ${ }^{3}$, Gilson F. da Silva ${ }^{4}$, Francinete V. Duarte ${ }^{1}$ \& Patrícia M. Neres ${ }^{1}$
}

\begin{abstract}
RESUMO
Este trabalho foi conduzido com o objetivo de verificar a ocorrência da associação da presença do carbofuran no perfil do solo com a forma e o tempo de aplicação do pesticida e, ainda, parâmetros de eficiência dos diversos sistemas de irrigação utilizados no Distrito de Irrigação do Gorutuba (DIG) em Janaúba, MG. Selecionaram-se 14 propriedades, que apresentaram grande variabilidade nos parâmetros de desempenho da irrigação e na distribuição do carbofuran no perfil do solo. Dos parâmetros analisados, o TAP (tempo de aplicação do produto), a QIAAP (quantidade do ingrediente ativo aplicado) e o Perc (Lâmina de irrigação percolada), apresentaram nítida associação com a distribuição do pesticida no perfil do solo. Esses parâmetros mostraram que produtores rurais dentro do DIG, os quais aplicam água em excesso via irrigação mal manejada e utilizam pesticidas de forma indiscriminada, não respeitando as recomendações técnicas nem o período de carência entre as aplicações, podem estar poluindo seriamente o solo e, em conseqüecia a água, comprometendo, a médio prazo, a sustentabilidade de suas atividades.
\end{abstract}

Palavras chave: transporte de pesticidas-solo, cultura da bananeira, eficiência de irrigação, contaminação-solo

\section{Assessment of carbofuran contamination in soil of the Irrigation District of Gorutuba}

\begin{abstract}
The work was carried out with the objective of verifying the association of carbofuran presence in the soil profile analyzing both form and time of application with efficiency parameters of irrigation systems used in the District of Irrigation of Gorutuba (DIG) in Janaúba, MG. Fourteen rural properties were selected that presented a great variability in the performance of irrigation parameters and in the distribution of the carbofuran in the soil profile. The parameters TAP (Time of application of the product), QIAAP (amount of the applied active ingredient) and Perc (Depth of percolated water), presented a clear association with the distribution of the pesticide in the soil profile. These parameters showed that farmers of the DIG, who apply excess water through irrigation and have bad management and also use pesticide indiscriminately may seriously pollute the soil and, consequently, the water, compromising in the medium term the sustainability of their activities.
\end{abstract}

Key words: pesticide-soil transport, banana, irrigation eficiency, soil-contamination

\footnotetext{
1 Instituto de Ciências Agrárias, Campus Regional de Montes Claros/UFMG, Av. Universitária, 1000, Bairro Universitário, CP 135, CEP 39404-006, Montes Claros, MG. Fone: (38) 2101-7708 Fax: (38) 2101-7703 E-mail: eovieira@nca.ufmg.br; Fone: (38) 2101-7723 Fax: (38) 2101-7703 E-mail: fveloso@nca.ufmg.br;

2 EMBRAPA - Centro Nacional de Pesquisa Milho e Sorgo, CP 151, CEP 35701-970 Sete Lagoas, MG. Fone: (31) 3027-1100. E-mail: htprates@cnpms.embrapa.br

${ }^{3}$ CEFET, BR 235, km 22, PSNC 04, Zona Rural, CP 178, CEP 56300-000, Petrolina, PE.

${ }^{4}$ Centro de Ciências Agrárias/UFES, Alto Universitário, CEP 16295-000, Alegre, ES. Fone: (28) 3558-1565. E-mail: gfsilva@cca.ufes.br
} 


\section{INTRODUÇÃO}

No Norte de Minas Gerais a fruticultura se localiza entre os municípios de Montes Claros, Capitão Enéas, Janaúba, Nova Porteirinha, Porteirinha, Jaíba, Manga, Januária e áreas circunvizinhas; sua área é de cerca de 16 mil ha, dos quais 73\% correspondem ao plantio da banana irrigada. Dentre os municípios citados, Janaúba se destaca como a capital desse pólo fruticultor em que está inserido o projeto de irrigação do Vale do Gorutuba, da Companhia de Desenvolvimento do Vale do São Francisco (CODEVASF, 2003), com área total de 7.064 ha, com 4.818 ha irrigáveis.

Com grande impulso em 1995 e retorno econômico rápido e satisfatório, a fruticultura no Norte de Minas teve expansão vertiginosa, juntamente com a irrigação, levando pecuaristas e muitos profissionais liberais da região a entrarem na atividade. A falta de conhecimento mais específico dessa nova atividade ou de uma assistência técnica adequada acarretou problemas relativos ao aspecto fitossanitário devido, sobretudo ao uso indiscriminado de pesticidas, especialmente nematicidas e inseticidas, em particular o carbofuran.

Dentre os pesticidas utilizados no projeto Gorutuba o carbofuran não é apenas um dos princípios ativos mas, também um dos mais utilizados no controle de nematóides e pragas, o qual, se aplicado de forma indiscriminada, vai de encontro aos prejuízos que podem estar sendo causados à natureza e ao próprio homem. Embora este princípio ativo não venha sendo recomendado por técnicos e agrônomos, produtores rurais da região insistem no seu uso devido à relativa eficiência no controle de nematóides, um patógeno que acarreta muitos problemas ao cultivo da bananeira.

O carbofuran, quando aplicado em solos de textura leve e irrigação de baixa eficiência, pode estar sendo lixiviado para camadas mais profundas do solo, podendo atingir lençóis freáticos.

O uso intensivo de pesticidas, o tipo de aplicação, toxicidade do composto usado, sistemas de irrigação de baixa eficiência em plantações de banana, são fatores que fazem do solo e do ecossistema aquático os mais vulneráveis tornando-se, assim, uma ameaça aos organismos e populações que habitam a região (Castillo et al., 2000).

Aliando a eficiência de irrigação ao uso indiscriminado do carbofuran no solo, conclui-se que diversos problemas podem estar sendo gerados, principalmente no que diz respeito à contaminação do solo e águas superficiais e subsuperficiais do perímetro irrigado; desta forma, o carbofuran poderá trazer problemas relacionados ao consumo dessa água pelas comunidades vizinhas e até mesmo ao meio ambiente, passível de distúrbios à saúde, não só ao agricultor envolvido diretamente na atividade mas a toda a sua família, afetando a sustentabilidade da atividade na região.

Tendo em vista o exposto propôs-se, neste trabalho, verificar a ocorrência da associação da presença do carbofuran em diversas camadas do solo com a forma e tempo de aplicação do pesticida e parâmetros de eficiência dos diversos sistemas de irrigação utilizados no Distrito de Irrigação do Gorutuba (DIG) em Janaúba, MG.

\section{MATERIAL E MÉTODOS}

O estudo consistiu em uma fase inicial com o levantamento, por meio de um questionário, das propriedades em que se utilizava o carbofuran; em uma segunda fase, de amostragem de solo e de avaliação do sistema de irrigação utilizado na propriedade e, na última fase, de análise físico-química em laboratório.

As propriedades avaliadas foram selecionadas com base nas respostas de produtores do perímetro irrigado do Gorutuba aos questionários aplicados na fase inicial dos trabalhos em campo, em que as áreas escolhidas ficaram restritas àquelas em que, segundo o proprietário, fôra aplicado o carbofuran em período máximo de doze meses.

Em cada propriedade foram amostrados três pontos (todos georreferenciados), e coletados em cada ponto, amostras de solo em camadas de $20 \mathrm{em} 20 \mathrm{~cm}$ até a profundidade de $100 \mathrm{~cm}$ utilizando-se trado tipo holandês; depois, amostras foram retiradas para caracterização física e química (análise textural, capacidade de campo, densidade aparente, umidade, análise química e matéria orgânica do solo) e análise de resíduo do carbofuran.

As amostras foram devidamente identificadas e embaladas com sacolas pretas e só então acondicionadas em freezer a $-5{ }^{\circ} \mathrm{C}$ logo que chegaram ao Laboratório de Química do Instituto de Ciências Agrárias (ICA), onde as análises físicas e químicas foram realizadas. A utilização de sacolas pretas fez-se necessário para evitar a fotodegradação de possíveis resíduos do princípio ativo existentes nas amostras no período de armazenamento.

Fex-se a determinação da capacidade de campo foi feita em mesa de tensão; por outro lado, a análise de resíduo foi dividida em duas etapas, ou seja, a extração foi realizada no Laboratório de Química do ICA e a quantificação dos resíduos no Laboratório de Agroquímica da Embrapa/Milho e Sorgo.

Depois de coletadas as amostras de solo, iniciaram-se os trabalhos de avaliação dos sistemas de irrigação da propriedade, encontrando-se o sistema de irrigação por aspersão subcopa, miniaspersão e microaspersão. Escolheram-se, para avaliação da uniformidade dos sistemas, três pontos de testes entre duas linhas laterais, localizadas no início, meio e fim da linha principal para o sistema de irrigação subcopa e miniaspersão e em linhas laterais situadas no início meio e fim das linhas de derivação para os sistemas de irrigações por microaspersão.

Realizaram-se avaliação dos sistemas com parâmetros de uniformidade de distribuição, e eficiência de irrigação, obtidos por meio de levantamento das características do sistema utilizado, tais como vazão e pressão de funcionamento dos emissores e pela precipitação de água dos emissores através de uma malha de coletores espaçados 0,5 m. A vazão de cada emissor foi determinada por meio da coleta do volume em um recipiente com tempo preestabelecido de 2 min; após este procedimento mediu-se o volume coletado utilizando-se uma proveta de capacidade de $2000 \mathrm{~mL}$; as pressões foram medidas nos mesmos pontos de medição de vazão, com a utilização de um manômetro de Bourdon e tubo Pitot, o qual era inserido no bocal de saída de água. 
Como a cultura da bananeira utiliza o sistema de um emissor para mais de uma planta se justifica, segundo Almeida (1997), o cálculo da uniformidade, através da distribuição de coletores em torno dos microaspersores.

Para os sistemas de irrigação subcopa e miniaspersores foram determinados o coeficiente de uniformidade de Christiansen (CUC), o coeficiente de uniformidade de distribuição (CUD) e o coeficiente de uniformidade estático (CUE) com dados coletados por meio da malha de coletores distribuídos entre quatro aspersores (Bernardo, 2005; Keller \& Bliesner, 1990).

A avaliação dos sistemas de irrigação por microaspersão em campo foi realizada de duas formas: pela determinação do coeficiente de uniformidade por meio da medição de vazão dos emissores ao longo da linha lateral e a outra com a coleta da precipitação de água dos emissores utilizando-se uma malha de pluviômetros eqüidistantes 0,5 m; esta segunda forma é sugerida por Almeida (1997) e Quaresma Filho (1999), visto que um microaspersor irriga quatro plantas necessitando, assim, avaliar a distribuição espacial da lâmina em torno do mesmo.

A uniformidade de aplicação de água por meio da medição de vazão foi calculada através do coeficiente de uniformidade de Christiansen para o sistema (Eq. 1).

$$
\mathrm{CUC}_{\text {sistema }}=100\left(1-\frac{\sum_{\mathrm{i}}^{\mathrm{n}}|\mathrm{Qi}-\overline{\mathrm{Q}}|}{\mathrm{n} \overline{\mathrm{Q}}}\right)
$$

em que:

$$
\begin{aligned}
\text { CUC }_{\text {sistema }} & \text { coeficiente de uniformidade de Christiansen para } \\
& \text { o sistema, \% } \\
\mathrm{Q} i \text { - } & \text { vazão coletada em cada emissor, } \mathrm{L} \mathrm{h}^{-1} \\
\overline{\mathrm{Q}} \text { - } & \text { média das vazões coletadas de todos os emis- } \\
& \text { sores, } \mathrm{L} \mathrm{h} \mathrm{h}^{-1} \\
\mathrm{~N}- & \text { número de emissores analisados }
\end{aligned}
$$

Para os procedimentos de cálculo dos coeficientes e classificação dos sistemas, utilizou-se o software Avalia 1.0, o qual fornece resultados de porcentagem de área adequadamente irrigada, perdas por percolação, eficiência de aplicação e gráfico de distribuição de lâminas na área.

\section{Extração e análise do resíduo de carbofuran em amostras de solo}

Antes de se iniciar a extração do resíduo de carbofuran do solo as amostras foram descongeladas e em seguida peneiradas em malha de $2 \mathrm{~mm}$, para remoção das pedras, raízes e material mais grosseiro, e posteriormente retirados $50 \mathrm{~g}$ (peso seco); para as análises de resíduo utiilizou-se o cromatógrafo líquido (HPLC), marca Shimadzu, modelo Class-LC10, com detector de ultravioleta, modelo SPD-10A e coluna C-18, modelo Zorbax ODS (4,6 mm x $25 \mathrm{~cm}$ ), marca DuPont, com porosidade de $5 \mathrm{~mm}$.

Para a extração e quantificação do carbofuran em solo pelo HPLC, utilizaram-se os parâmetros recomendados por Vieira et al. (2001) adaptados às condições do laboratório de agroquímica da EMBRAPA de Sete Lagoas.

\section{RESULTADOS E DISCUSSÃO}

\section{Análise dos parâmetros levantados}

De acordo com o questionário realizado na primeira fase do experimento, 14 propriedades foram escolhidas incluindo-se a margem esquerda e o Distrito de Irrigação do Gorutuba (DIG), Figura 1.

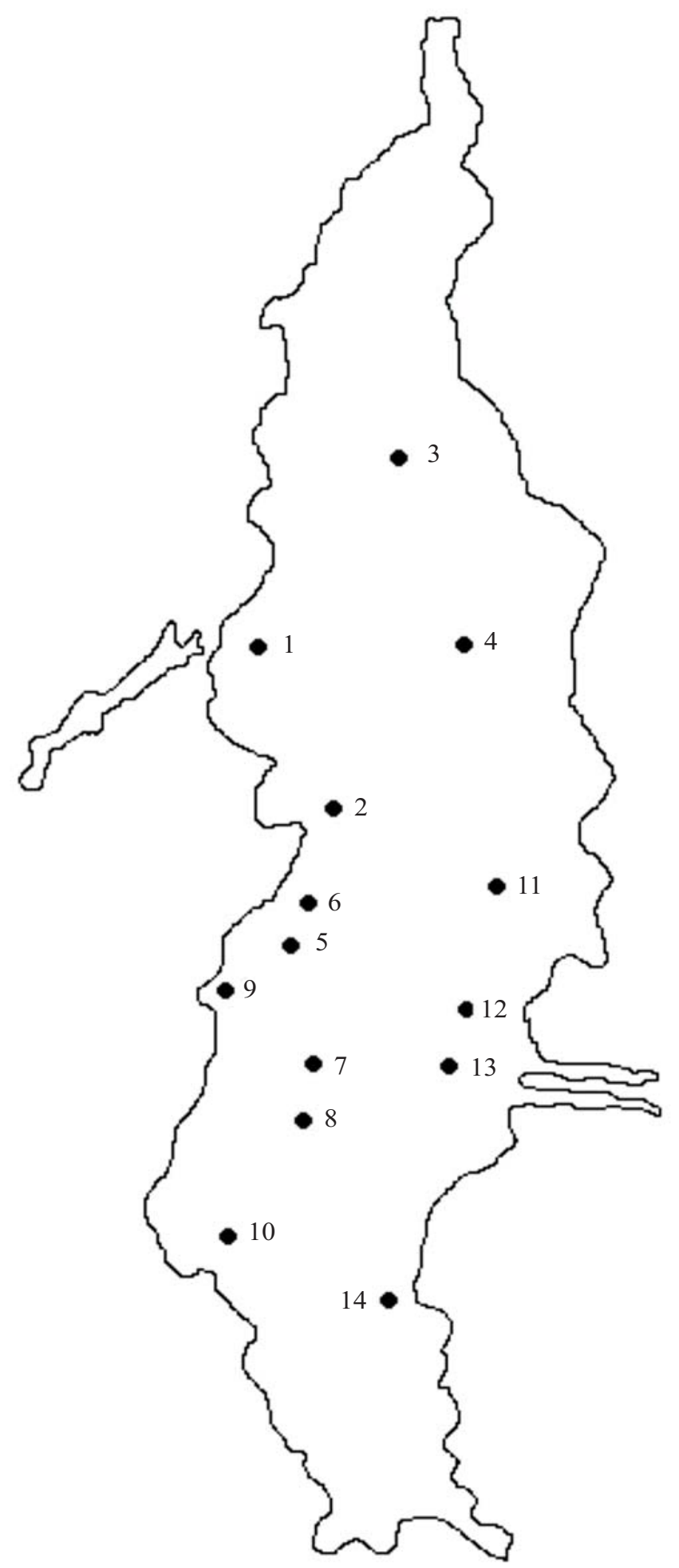

Figura 1. Projeto de irrigação do Gorutuba e localização dos 14 pontos de amostragem georreferenciados 
Os parâmetros físico-hídricos dos solos avaliados referentes à análise textural capacidade de campo e densidade do solo e teor de matéria orgânica, são apresentados na Tabela 1. Devido a classificação textural dos solos avaliados se ter enquadrado, em sua maioria, entre franco a franco-arenoso, utilizou-se a mesa de tensão com $0,1 \mathrm{kPa}$ para determinação da umidade correspondente à capacidade de campo. Os resultados apresentados nesta Tabela 1 se referem aos valores médios das três primeiras camadas amostradas, ou seja, de 0-60 cm os quais possuem maior importância agronômica para o cultivo da banana.

Tabela 1. Características físico-hídricas e teor de matéria orgânica dos solos amostrados

\begin{tabular}{|c|c|c|c|c|}
\hline Lote & Classificação Textural & $\begin{array}{c}\text { Capac. Campo } \\
\text { (\%) }\end{array}$ & $\begin{array}{l}\text { Dens. Solo } \\
\left(\mathrm{g} \mathrm{cm}^{-3}\right)\end{array}$ & $\begin{array}{l}\text { MO } \\
(\%)\end{array}$ \\
\hline 1 & franco arenoso & 17,5 & 1,56 & 1,01 \\
\hline 2 & franco argilo arenoso & 19,5 & 1,35 & 1,11 \\
\hline 3 & franco-argiloso & 24 & 1,10 & 3,25 \\
\hline 4 & franco & 27 & 1,35 & 3,71 \\
\hline 5 & franco & 22 & 1,43 & 2,43 \\
\hline 6 & franco siltoso & 14 & 1,32 & 2,10 \\
\hline 7 & franco siltoso & 16 & 1,29 & 2,97 \\
\hline 8 & franco argiloso & 24 & 1,19 & 3,95 \\
\hline 9 & franco arenoso & 18,1 & 1,56 & 0,83 \\
\hline 10 & franco siltoso & 16,9 & 1,31 & 3,12 \\
\hline 11 & franco siltoso & 15 & 1,27 & 2,41 \\
\hline 12 & franco argiloso & 21,3 & 1,13 & 3,07 \\
\hline 13 & franco arenoso & 18,3 & 1,45 & 0,91 \\
\hline 14 & franco arenoso & 17,2 & 1,54 & 0,74 \\
\hline
\end{tabular}

Verifica-se, na Tabela 1, que os lotes amostrados se enquadram dentro dos solos caracterizados como aluviais arenosos, aluviais francos e aluviais argilosos, conforme o levantamento de aptidão para irrigação e mapeamento pedológico apresentado pela CODEVASF (1978). Os valores de densidade do solo acima de $1,5 \mathrm{~g} \mathrm{~cm}^{-3}$, podem estar relacionados a solos compactados, resultado do uso intensivo e manejo inadequado do solo, enquanto na Tabela 2 se apresentam algumas informações obtidas por meio do questionário aplicado aos produtores rurais.

Na Tabela 3, se observa que os coeficientes de uniformidade de Christiansen apresentaram variabilidade muito alta, com valores de 2,78 a 95,24\% sendo que dos 14 lotes avaliados 21\% foram classificados excelentes; $43 \%$ bons; $7 \%$ foram razoáveis e 29\%, inadequados; já o coeficiente de uniformidade de distribuição classificou 29\% dos lotes excelentes; 43\% bons; 7\% razoáveis e 21\% inaceitáveis.

Segundo Almeida (1997), o CUC pode não retratar a realidade da uniformidade de aplicação de água porque na irrigação de culturas como a da banana, que utilizam sistemas por aspersão subcopa e miniaspersão, os pseudo-caules interceptam a água aspergida, que fica à disposição das plantas mas não são coletadas nos pluviômetros; isto pode ser verificado na Tabela 3, na qual os menores valores de CUC e CUD foram apresentados, em sua grande maioria, pelos sistemas de irrigação subcopa e miniaspersão.
Tabela 2. Caracterização quanto ao sistema de irrigação utilizado e à aplicação do carbofuran

\begin{tabular}{cllcc}
\hline Lote & Tipo Irrigação & Forma de aplicação do Produto & $\begin{array}{c}\text { QIAAP } \\
\text { (g) }\end{array}$ & $\begin{array}{c}\text { TAP } \\
\text { (meses) }\end{array}$ \\
1 & Microaspersão & $10 \mathrm{~g}$ por cova & 0,5 & 12 \\
2 & Microaspersão & $300 \mathrm{~mL}$ por cova & 122,5 & 9 \\
3 & Miniaspersão & $10 \mathrm{~g}$ por cova & 0,5 & 7 \\
4 & Microaspersão & $20 \mathrm{~g}$ por pé & 1,0 & 12 \\
5 & Sub-Copa & $250 \mathrm{~mL}$ por cova & 87,5 & 6 \\
6 & Miniaspersão & $5 \mathrm{~g}$ por toco com lurdinha & 70,25 & 4,5 \\
7 & Microaspersão & $200 \mathrm{~mL}$ pl$^{-1}$ e $300 \mathrm{~mL}$ por cova & 122,5 & 12 \\
8 & Microaspersão & $20 \mathrm{~g}$ por pé & 1 & 3 \\
9 & Miniaspersão & $15 \mathrm{~g}$ por pé e $5 \mathrm{~g}$ por toco & 1 & 8 \\
10 & Miniaspersão & $15 \mathrm{~g}$ por pé e $5 \mathrm{~g}$ por toco & 1 & 8 \\
11 & Microaspersão & $10 \mathrm{~g}$ por cova & 0,5 & 12 \\
12 & Sub-Copa & $5 \mathrm{~g}$ por toco e $250 \mathrm{~mL}$ por cova & 87,75 & 3 \\
13 & Microaspersão & $20 \mathrm{~g}$ por pé & 1 & 4 \\
14 & Miniaspersão & $5 \mathrm{~g}$ por toco e $5 \mathrm{~g}$ por pé & 0,5 & 3 \\
\hline
\end{tabular}

QIAAP - quantidade do ingrediente ativo aplicado; TAP - Tempo decorrido da aplicação do produto até a retirada das amostras de solo

Tabela 3. Valores dos parâmetros de desempenho da irrigação para os lotes avaliados no projeto Gorutuba, MG

\begin{tabular}{clcccccc}
\hline Lote & Tipo Irrigação & $\begin{array}{c}\text { CUC } \\
\mathbf{( \% )}\end{array}$ & $\begin{array}{c}\text { CUD } \\
(\mathbf{\%})\end{array}$ & $\begin{array}{c}\text { CUEa } \\
(\mathbf{\%})\end{array}$ & $\begin{array}{c}\text { CUE } \\
\mathbf{( \% )}\end{array}$ & $\begin{array}{c}\text { Perc } \\
(\mathbf{m m})\end{array}$ & $\begin{array}{c}\text { Ea } \\
(\%)\end{array}$ \\
1 & Microasp. & 82,21 & 74,20 & 73,18 & 81,26 & 7,87 & 42,64 \\
2 & Microasp. & 87,89 & 77,81 & 81,51 & 83,51 & 4,95 & 60,55 \\
3 & Miniasp. & 51,39 & 36,67 & - & - & 0,36 & 61,01 \\
4 & Microasp. & 91,66 & 84,54 & 86,54 & 88,36 & 1,28 & 91,40 \\
5 & Sub-Copa & 91,03 & 87,99 & - & - & 38,77 & 81,86 \\
6 & Miniasp. & 82,09 & 76,41 & 72,76 & 82,50 & 0,00 & 70,61 \\
7 & Microasp. & 95,24 & 92,54 & 93,68 & 94,13 & 0,00 & 100,00 \\
8 & Microasp. & 78,33 & 70,46 & 70,06 & 78,21 & 0,00 & 100,00 \\
9 & Miniasp. & 23,85 & 16,25 & - & - & 2,74 & 42,14 \\
10 & Miniasp. & 2,78 & 14,56 & & & 5,68 & 36,88 \\
11 & Microasp. & 86,39 & 86,11 & 76,93 & 89,49 & 1,11 & 91,59 \\
12 & Sub-Copa & 26,17 & 26,87 & - & - & 5,46 & 57,99 \\
13 & Microasp. & 89,88 & 81,28 & 84,33 & 86,01 & 4,70 & 64,94 \\
14 & Miniasp. & 86,59 & 83,54 & 79,24 & 87,64 & 9,92 & 42,70 \\
\hline
\end{tabular}

CUC - Coeficiente de uniformidade de Christiansen; CUD - Coeficiente de uniformidade de distribuição; CUEa - Coeficiente de uniformidade de emissão absoluta; CUE - Coeficiente de uniformidade estática; Perc - Percolação; Ea - Eficiência de aplicação

\section{Análise de resíduo de carbofuran no solo}

As Figuras 2 e 3 mostram a variação da concentração do resíduo de carbofuran com a profundidade do solo. Os resultados foram apresentados apenas nos lotes 2, 3 e 5 (Figura 2) e nos lotes 12,13 e 14 (Figura 3), nos quais havia resíduos de carbofuran; nos demais pontos amostrados não se detectaram resíduos de carbofuran.

$\mathrm{Na}$ faixa de $\mathrm{pH}$ mais comum dos solos tropicais agricultáveis (5,0 a 6,5, na qual $\mathrm{pH}>\mathrm{pKa}$ ), como os solos do projeto Gorutuba, os pesticidas que manifestam caráter ácido no solo podem apresentar carga negativa em virtude da sua dissociação na forma aniônica ocorrendo, assim uma baixa adsorção aos colóides do solo, devido às forças eletrostáticas repulsivas com a carga líquida negativa dos solos com alto grau de intemperismo, motivo por que os pesticidas ácidos são normalmente menos sorvidos aos colóides do solo que os pesticidas neutros ou básicos. Quando o pH da solução 


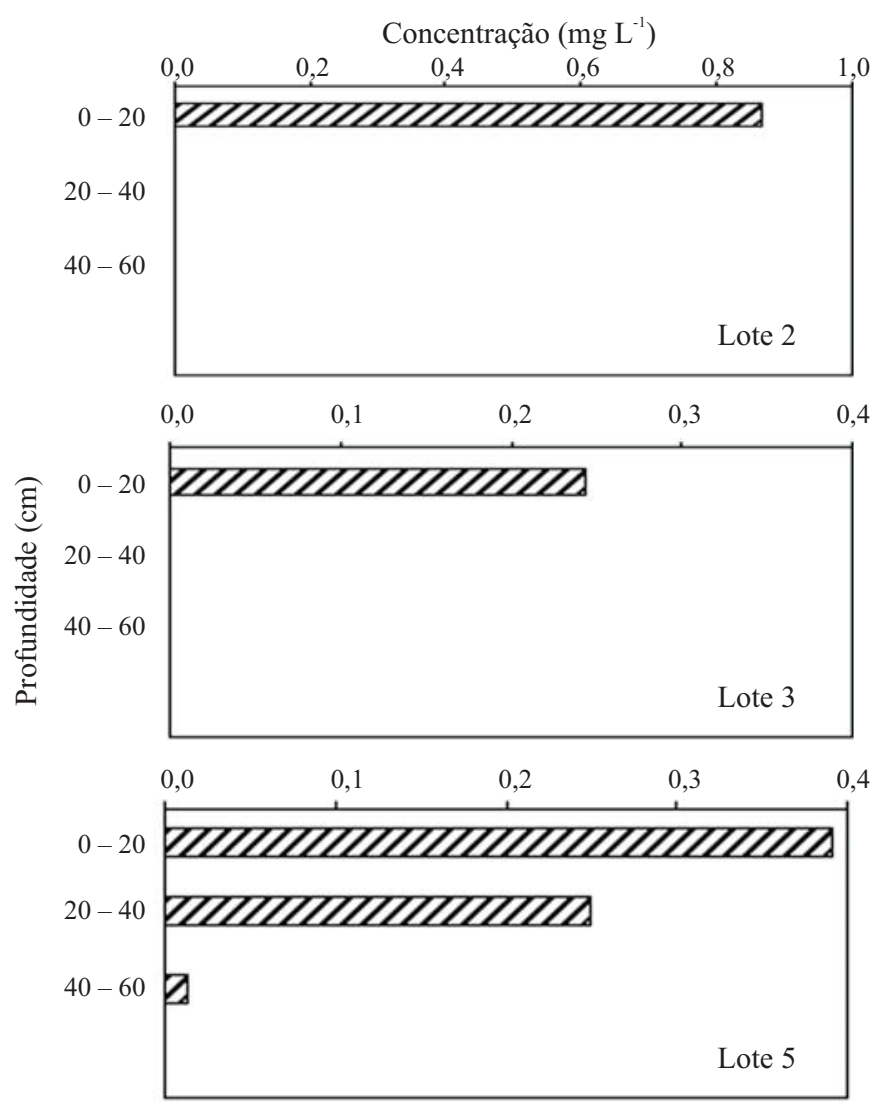

Figura 2. Distribuição do carbofuran no perfil do solo dos lotes amostrados (2, 3 e 5$)$

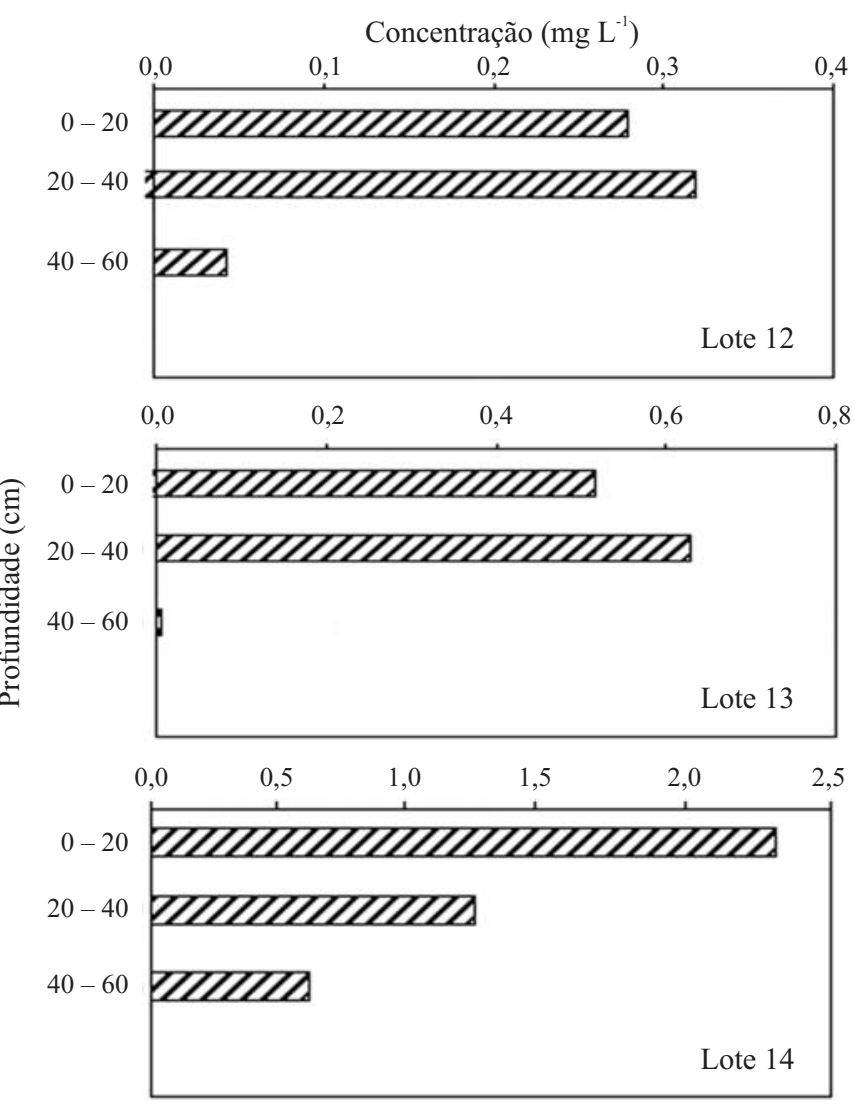

Figura 3. Distribuição do carbofuran no perfil do solo dos lotes amostrados $(12,13$ e 14) do solo está próximo ao pKa do pesticida, ocorre protonação do carbofuran, que passa para a forma neutra; nesta condição, as interações hidrofóbicas com a matéria orgânica do solo passam a predominar e a sorção aumenta porém, em razão dos baixos valores de matéria orgânica apresentados pela maioria dos solos amostrados, associados a um $\mathrm{pH}$ possivelmente ácido, comum aos solos brasileiros, o carbofuran apresentou certa facilidade no seu deslocamento ao longo do perfil do solo em alguns lotes, em que outros fatores também se mostraram favoráveis a este processo.

\section{Avaliação da associação dos parâmetros levantados com a distribuição do carbofuran no solo}

A partir das variáveis medidas nos 14 lotes, verifica-se que o movimento do produto testado no solo ocorreu de forma diferenciada podendo-se sugerir, a priori, a formação de 3 grupos distintos entre si, ou seja, um primeiro grupo em que o produto não se movimentou no solo, isto é, não foram detectados resíduos em nenhuma das profundidades analisadas; este grupo é composto dos 1, 4, 6, 7, 8, 9, 10 e 11; um segundo grupo, em que foi pouca a movimentação do produto no solo e no qual se detectaram resíduos apenas na camada de $0-20 \mathrm{~cm}$, formado dos lotes 2 e 3 e um terceiro grupo, no qual ocorreu uma movimentação maior do produto no solo, ou seja, nos em que foram detectados resíduos de carbofuran até $60 \mathrm{~cm}$ de profundidade; este grupo se formava dos lotes 5, 12, 13 e 14 .

Realizou-se a divisão prévia dos grupos, o próximo passo foi verificar se esses grupos sugeridos se apresentavam consistentes e quais as variáveis que mais contribuíram para a sua formação; para tanto, foi implementada uma análise discriminante em que os casos foram os lotes já citados e as variáveis aquelas já apresentadas nas Tabelas 2 e 3. Os parâmetros de desempenho CUEa e CUE não foram considerados nesta análise devido à impossibilidade de seu cálculo para todos os lotes avaliados; a análise teve como objetivo verificar se os grupos sugeridos foram discriminados corretamente e, com auxílio do procedimento Forward para funções discriminantes, utilizando-se a estatística $\mathrm{F}$, identificar as variáveis que mais contribuíram para a formação dos grupos propostos a priori. Todas as análises foram feitas utilizando-se o programa Statistica 6.0 de propriedade da empresa StatSoft.

Após realizar o procedimento Forward para funções discriminantes no programa Statistica, chegou-se aos seguintes resultados, conforme apresentado na Tabela 4.

Tabela 4. Estatísticas das variáveis selecionadas e não selecionadas pelo procedimento Forward para formação da função discriminante

\begin{tabular}{|c|c|c|c|c|c|}
\hline $\begin{array}{l}\text { Variáveis } \\
\text { Selec. }\end{array}$ & $\begin{array}{l}\text { Wilks' } \\
\text { Lambda }\end{array}$ & $\begin{array}{c}\text { Lambda } \\
\text { Parcial }\end{array}$ & F aprox. & Prob. & Tolerância \\
\hline TAP & 0,02768 & 0,43439 & 3,91 & 0,082 & 0,36 \\
\hline PERC & 0,03149 & 0,38185 & 4,86 & 0,056 & 0,57 \\
\hline QIAAP & 0,01646 & 0,73043 & 1,11 & 0,389 & 0,33 \\
\hline $\begin{array}{l}\text { Variáveis } \\
\text { Não Selec. }\end{array}$ & $\begin{array}{l}\text { Wilks' } \\
\text { Lambda }\end{array}$ & $\begin{array}{l}\text { Lambda } \\
\text { Parcial }\end{array}$ & F aprox. & Prob. & Tolerância \\
\hline CUC & 0,01107 & 0,92093 & 0,21 & 0,814 & 0,63 \\
\hline CUD & 0,01161 & 0,96578 & 0,09 & 0,917 & 0,61 \\
\hline $\mathrm{EA}$ & 0,01169 & 0,97227 & 0,07 & 0,932 & 0,15 \\
\hline
\end{tabular}

Prob. - nível de significância para 0 teste $\mathrm{F}$ 
Para facilitar a compreensão, as estatísticas apresentadas na Tabela 4 serão discutidas, uma a uma, procurando-se esclarecer o procedimento Forward de determinação da melhor função discriminante. Este procedimento procura identificar, também uma a uma as variáveis estatisticamente mais significantes para compor a função discriminante. O teste de Wilks’ Lambda é a estatística padrão usada para denotar a significância estatística do poder discriminatório da função discriminante em questão. $\mathrm{O}$ valor do teste pode variar de 0,0 a 1,0 considerando-se que, quanto mais próximo de 1,0 menor será o poder discriminatório da função, sendo o contrário verdadeiro; a segunda coluna da Tabela 4 mostra os valores do teste Wilks’Lambda após a respectiva variável ter entrado no modelo; já o Lambda Parcial é variação do teste de Wilks' Lambda que calcula a significância estatística isolada para cada variável, ou seja, informa o quanto cada variável é isoladamente importante para a função discriminatória; a interpretação é a mesma, isto é, quanto mais próximo o valor de 1,0 menor é a participação da variável na discriminação dos grupos, sendo o contrário verdadeiro. Deste ponto de vista e se considerando a Tabela 4, observam-se as variáveis que apresentaram menores valores de Lambda Parcial que, pela ordem, foram: Percolação (Perc) e Tempo de aplicação do produto (TAP), podendo-se dizer que elas foram, respectivamente, as variáveis mais importantes para discriminar os grupos; as variáveis Perc e TAP podem estar relacionadas com a formação dos grupos pelo fato de que a primeira variável está relacionada com o excesso de água aplicado pelo agricultor, o que, de forma direta, reflete a baixa eficiência do manejo de irrigação utilizado favorecendo para que produtos químicos de alta solubilidade e baixa reatividade no solo, possam atingir camadas mais profundas. O tempo de aplicação do produto (TAP), que reflete o tempo compreendido entre a aplicação do pesticida e a data da amostragem do solo para análise de resíduo, está relacionado com a meia vida do pesticida que, associado ao manejo da irrigação, pode refletir nos valores de resíduo encontrados nas diferentes camadas do solo; isto pode ser explicado de forma que, quanto maior o TAP, maior a quantidade do pesticida que pode ter sido degradado pelas diversas formas de reação no solo sendo elas físico, químicas e biológicas

Quando se observa os resultados da Tabela 4, nota-se também que, para as variáveis não selecionadas os valores de Lambda Parcial são muito próximos de 1,0, indicando que as variáveis CUC, CUD e EA não foram incluídas na função discriminante pelo procedimento Forward, visto que apresentam pouca significância estatística para discriminação dos grupos; isto ocorreu porque a distribuição do pesticida no solo não está, provavelmente, relacionada com a forma com que a água é aplicada e, sim, com a quantidade aplicada.

O teste de F é uma aproximação da estatística de Wilks’ Lambda pela estatística F padrão e também informa, tal como o Lambda Parcial, a importância isolada das variáveis para a função discriminante. Ao lado da estatística F tem-se na Tabela 4 o respectivo nível de significância dessa estatística; pode-se, então, observar que esta estatística tem interpretação semelhante àquela feita para o Lambda Parcial podendo-se observar, também na Tabela 4, que a variável QIAAP (quantidade do ingrediente ativo aplicado) foi pouco significativa pelo teste $\mathrm{F}$ quando se observa o seu nível de significância. A Tabela 4 mostra que, realmente, as variáveis possuem pouca significância estatística pelo teste F não devendo, de acordo com o procedimento Forward, participar do modelo de discriminação dos grupos.

Por fim, as colunas da Tabela 4, denominadas Tolerância, informam o quanto uma variável pode ser redundante com outras variáveis do modelo de discriminação. O valor de Tolerância de uma variável nada mais é do que 1 menos o $\mathrm{R}$ quadrado, em que $\mathrm{R}^{2}$ é a correlação múltipla para cada variável com outras variáveis que já estão presentemente incluídas no modelo. O valor de Tolerância obtido pela expressão $1-\mathrm{R}^{2}$, é uma medida da redundância de uma variável com outras já existentes no modelo de discriminação. A título de exemplo, se o valor de Tolerância for igual a 0,01 para uma variável qualquer, significa que a variável em questão pode ser considerada 99\% redundante com as variáveis já incluídas no modelo; variáveis muito redundantes, como no caso do exemplo dado, devem ser evitadas, pois podem causar problemas na matriz de variância/covariância das variáveis incluídas no modelo, comprometendo as análises da função discriminante; em relação às variáveis selecionadas para o modelo discriminante (Tabela 4), as variáveis TAP e QIAAP apresentaram menores valores de tolerância mas não são baixos o suficiente para que se pense em uma redundância que possa comprometer a análise.

Depois de implementado o procedimento Forward e se considerando todas as estatísticas apresentadas verifica-se, ao final, que as variáveis da Tabela 4 para variáveis não selecionadas, foram as escolhidas para a função discriminante dos grupos propostos, com um valor de $\mathrm{F}$ aproximado para o teste de Wilks’ Lambda significativo em nível de $1 \%$ de probabilidade. Procedeu-se, em seguida, a um exame de classificação dessa função discriminante e se observou que $100 \%$ dos casos foram classificados corretamente, ou seja, que os grupos propostos de inicio foram formados corretamente, não devendo haver mudanças nos mesmos, isto é, os lotes pertencentes aos grupos 1, 2 e 3 devem permanecer nos seus respectivos grupos.

\section{CONCLUSÕES}

1. Para o carbofuran aplicado no cultivo da banana, não houve contaminação extensiva do solo sendo que resíduos do defensivo foram encontrados a uma profundidade máxima de $60 \mathrm{~cm}$; mesmo assim, em apenas alguns lotes do total analisado.

2. Os parâmetros de desempenho do sistema de irrigação como CUD, CUC e EA não apresentaram associação satisfatória com a distribuição do carbofuran no perfil do solo.

3. Os parâmetros TAP (Tempo de aplicação do produto), QIAAP (quantidade do ingrediente ativo aplicado) e Perc (Lâmina de irrigação percolada), apresentaram nítida associação com a distribuição do pesticida no perfil do solo.

4. Os parâmetros acima relacionados mostraram que produtores rurais dentro do DIG, os quais aplicam excesso de água via irrigação mal manejada e utilizam pesticidas, cujo 
princípio ativo seja o carbofuran, de forma indiscriminada, não respeitam as recomendações técnicas nem o período de carência entre as aplicações e podem estar poluindo seriamente o solo e, conseqüentemente, a água comprometendo, a médio prazo a sustentabilidade de suas atividades.

\section{LITERATURA CITADA}

Almeida, F. T. Avaliação dos sistemas de irrigação pressurizados e do manejo da água na cultura da banana no Projeto Gorutuba. Viçosa: UFV, 1997. 100p. Dissertação Mestrado

Bernardo, S. Manual de irrigação. 8.ed. Viçosa: UFV, 2005. 657p.

Castillo, L. E.; Ruerpert, C.; Solis, E. Pesticide residues in the aquatic environment of banana plantations areas in the north atlantic zone of zone Costa Rica. Environmental Toxicology and Chemistry, v.19, n.8, p.1942-50, 2000.
CODEVASF - Companhia de Desenvolvimento do Vale do São Francisco. Levantamento de aptidão para irrigação e mapeamento pedológico. Brasília: 1978, 78p.

CODEVASF - Companhia de Desenvolvimento do Vale do São Francisco. O projeto Gorutuba. Brasília - DF, 2003. http://www.codevasf.gov.br/produtos/pro_gorutuba.htm. 28 Dez. 2005.

Keller, J.; Bliesner, R. D. Sprinkle and trickle irrigation. New York: Van Nostrand Reinhold, 1990. 652p.

Quaresma Filho, M. I. M. Avaliação dos sistemas de irrigação por microaspersão na fruticultura dos projetos Gorutuba e Jaíba Viçosa: UFV, 1999. 95p. Dissertação Mestrado

Vieira, E. O.; Prates, H. T.; Cruz, I.; Ramos, M. M.; Silva, G. F. Desenvolvimento de método simplificado de determinação de resíduos de carbofuran e thiodicarb em solo com a utilização de cromatografia líquida. Revista Ceres, v.280, n.148, p.659-670, 2001. 